\title{
EXISTENCE THEOREM FOR GROUPS
}

\author{
by C. G. CHEHATA \\ (Received 18th June 1963)
}

\section{Introduction}

Given a partial automorphism of a group $G$, i.e. an isomorphic mapping $\mu$ of a subgroup $A$ of $G$ onto a second subgroup $B$ of $G$, it is known (2, Theorem I) that there always exists a group $H$ containing $G$ and an inner automorphism of $H$ which extends $\mu$; i.e. there exists an element $t$ of $H$, such that the transform by $t$ of any element of $A$ is its image under $\mu$.

Also it is known (2, Theorem II) that this result generalises to the case in which $G$ possesses any number, finite or infinite, of partial automorphisms. These can be simultaneously extended to inner automorphisms of one and the same supergroup.

In (1) conditions are derived which are sufficient for extending two partial automorphisms of a given group to commutative automorphisms (inner ones) of an extension supergroup.

In this paper we consider a given group $G$ and a set of partial automorphisms $\mu(\sigma)$ of $G$, where $\sigma$ ranges over an index set $\Sigma$ and derive conditions which are sufficient for $\mu(\sigma)$ to be all extendable to inner automorphisms $t_{\sigma}$ of one and the same supergroup $G^{*} \supseteq G$ such that for a fixed $\alpha \in \Sigma, t_{\alpha}$ commutes with every $t_{\sigma}$. An obviously necessary condition for this is that $\mu(\alpha)$ commutes with every $\mu(\sigma), \sigma \in \Sigma$.

The sufficient conditions obtained, though not necessary, are wide enough to give Corollaries (1) and (2) as special cases.

\section{First step of the construction}

Let $A_{\sigma}$ and $B_{\sigma}$, where $\sigma$ ranges over an index set $\Sigma$, be subgroups of a given group $G$, and assume that for every $\sigma \in \Sigma, \mu_{\sigma}$ is an isomorphic mapping of $A_{\sigma}$ onto $B_{\sigma}$. Let $\propto$ be a fixed element of $\Sigma$, such that

$$
\begin{aligned}
& \left(A_{\alpha} \cap A_{\theta}\right) \mu_{\alpha}=B_{\alpha} \cap A_{\theta}, \\
& \left(A_{\alpha} \cap B_{\theta}\right) \mu_{\alpha}=B_{\alpha} \cap B_{\theta}, \\
& \left(A_{\alpha} \cap A_{\theta}\right) \mu_{\theta}=A_{\alpha} \cap B_{\theta}, \\
& \left(B_{\alpha} \cap A_{\theta}\right) \mu_{\theta}=B_{\alpha} \cap B_{\theta},
\end{aligned}
$$

for all $\theta(\neq \alpha) \in \Sigma$

$$
\mathrm{g} \mu_{\alpha} \mu_{\theta}=\mathrm{g} \mu_{\theta} \mu_{\alpha}
$$

for all $\theta \in \Sigma$ and whenever $g \mu_{\alpha}, g \mu_{\theta},\left(g \mu_{\alpha}\right) \mu_{\theta}$ and $\left(g \mu_{\theta}\right) \mu_{\alpha}$ are defined. 
Take a sequence of groups

$$
\ldots, G^{(-1)}, G^{(0)}, G^{(1)}, G^{(2)}, \ldots
$$

which are copies of the group $G$, i.e. each group $G^{(i)}, i=0, \pm 1, \pm 2, \ldots$ is isomorphic to $G$ under a fixed isomorphic mapping $\gamma^{i}$ :

$$
G^{(i)}=G^{\gamma^{i}} \text {. }
$$

Lemma. $G^{(i)}$ contains, for every $\sigma \in \Sigma$, subgroups $A_{\sigma}^{(i)}$ and $B_{\sigma}^{(i)}$ which are isomorphic.

Proof. Each group $G^{(i)}$ contains, for every $\sigma \in \Sigma$, subgroups $A_{\sigma}^{(i)}$ and $B_{\sigma}^{(i)}$ which are images of $A_{\sigma}, B_{\sigma}$ respectively under the mapping $\gamma^{i}$. Let $\mu_{\sigma}^{i}$ be the mapping defined as follows. If

and if

$$
\begin{array}{ll}
a_{\sigma}^{(i)}=a_{\sigma} \gamma^{i}, & a_{\sigma} \in A_{\sigma}, \\
b_{\sigma}^{(i)}=b_{\sigma} \gamma^{i}, & b_{\sigma} \in B_{\sigma}
\end{array}
$$

then we put

$$
a_{\sigma} \mu_{\sigma}=b_{\sigma}
$$

This is the mapping,

$$
a_{\sigma}^{(i)} \mu_{\sigma}^{i}=b_{\sigma}^{(i)}
$$

$$
\mu_{\sigma}^{i}=\left(\gamma^{i}\right)^{-1} \mu_{\sigma} \gamma^{i}
$$

$\mu_{\sigma}^{i}$ is an isomorphism of $A_{\sigma}^{i}$ onto $B_{\sigma}^{i}$.

For any two integers $i, j$ such that $i<j$, we define by induction a sequence of groups $P^{i, j}$ as follows.

We first form the free product of $G^{(i)}$ and $G^{(i+1)}$ amalgamating $B_{\alpha}^{(i)} \subseteq G^{(i)}$ with $A_{a}^{(i+1)} \subseteq G^{(i+1)}$ by putting $b_{a}^{(i)}=a_{a}^{(i+1)}$ whenever

and

$$
\begin{gathered}
b_{\alpha}^{(i)}=b_{\alpha} \gamma^{i}, \quad b_{\alpha} \in B_{\alpha}, \\
a_{\alpha}^{(i+1)}=a_{\alpha} \gamma^{i+1}, \quad a_{\alpha} \in A_{\alpha}
\end{gathered}
$$

$$
a_{\alpha} \mu_{\alpha}=b_{\alpha} \text {. }
$$

Thus the isomorphism underlying the amalgamation is $\left(\gamma^{i}\right)^{-1} \mu_{\alpha} \gamma^{i+1}$. This mapping is defined on $B_{\alpha}^{(i)}$; moreover it is the identical mapping on $B_{\alpha}^{(i)}$.

Let

and inductively

$$
P^{i, i+1}=\left\{G^{(i)} * G^{(i+1)} ; B_{\alpha}^{(i)}=A_{a}^{(i+1}\right\},
$$

$$
P^{i, j}=\left\{P^{i, j-1} * G^{(j)} ; B_{a}^{(j-1)}=A_{a}^{(j)}\right\} .
$$

We then form the union

$$
P^{*}=\bigcup_{n=1}^{\infty} P^{-n,+n}
$$

and define the mapping $\mu_{\alpha}^{*}$ as follows. For any $x \in P^{*}$, i.e. $x \in G^{(i)}$ for some suitable $i$, let $x\left(\gamma^{i}\right)^{-1}=g \in G, g \gamma^{i+1}=y$; then we put

$$
x \mu_{\alpha}^{*}=y,
$$


which means that on $G^{(i)}, \mu_{\alpha}^{*}$ is the mapping $\left(\gamma^{i}\right)^{-1} \gamma^{i+1}$.

Now we have the following result.

Theorem 1. The group $G$ is embedded in the supergroup $P^{*} \supseteq G$ which possesses an automorphism $\mu_{\alpha}^{*}$ extending $\mu_{\alpha}$ and for every $\theta(\neq \alpha) \in \Sigma, P^{*}$ contains the subgroups

and

$$
A_{\theta}^{*}=\left\{\ldots, A_{\theta}^{(-1)}, A_{\theta}^{(0)}, A_{\theta}^{(1)}, \ldots\right\}
$$

$$
B_{\theta}^{*}=\left\{\ldots, B_{\theta}^{(-1)}, B_{\theta}^{(0)}, B_{\theta}^{(1)}, \ldots\right\}
$$

which are isomorphic under a mapping $\bar{\mu}_{\theta}$ such that for any $x \in A_{\theta}^{*}$,

$$
x \bar{\mu}_{\theta} \mu_{\alpha}^{*}=x \mu_{\alpha}^{*} \bar{\mu}_{\theta} .
$$

The proof of this theorem follows the same lines as that of lemmas 5-9 in (1). What is true there for $\mu$ holds here for $\mu_{\alpha}$ and what is true there for $v$ holds here for every $\mu_{\theta}, \theta(\neq \alpha) \in \Sigma$.

\section{Second step of the construction}

Now we form the group

$$
\widetilde{P}=\left\{P^{*}, t_{\alpha}\right\}
$$

generated by $P^{*}$ and an element $t_{\alpha}$ and define

$$
t_{\alpha}^{-1} p^{*} t_{\alpha}=p^{*} \mu_{\alpha}^{*}
$$

for all $p^{*} \in P^{*}$.

Thus $t_{\alpha}$ induces an inner automorphism of $P$ and equation (6) gives

$$
t_{\alpha}^{-1}\left(x \bar{\mu}_{\theta}\right) t_{\alpha}=\left(t_{\alpha}^{-1} x t_{\alpha}\right) \bar{\mu}_{\theta}
$$

which shows that the inner automorphism induced by $t_{\alpha}$ commutes with every $\bar{\mu}_{\theta}, \theta(\neq \alpha) \in \Sigma$.

For every $\theta(\neq \alpha) \in \Sigma$, define

$$
t_{\alpha} \bar{\mu}_{\theta}=t_{\alpha},
$$

thus $\bar{\mu}_{\theta}$ becomes an isomorphism of

onto the group

$$
\tilde{A}_{\theta}=\left\{A_{\theta}^{*}, t_{\alpha}\right\}
$$

$$
\widetilde{B}_{\theta}=\left\{B_{\theta}^{*}, t_{\alpha}\right\}
$$

which also commutes with the inner automorphism induced by $t_{\alpha}$.

Applying Theorem II, (2) we can embed $G$ in a group $G^{*}$ containing a group $T$ freely generated by a set of elements $t_{\theta}, \theta(\neq \alpha) \in \Sigma$, such that for any $\theta(\neq \alpha)$ in $\Sigma$ the transform by $t_{\theta}$ of an element in $A_{\theta}$ is its image $\bar{\mu}_{\theta}$, i.e.

$$
t_{\theta}^{-1} \tilde{a}_{\theta} t_{\theta}=\tilde{a}_{\theta} \bar{\mu}_{\theta}
$$

for any $\tilde{a}_{\theta} \in \tilde{A}_{\theta}$.

This means that $t_{\theta}$ induces an inner automorphism of $G^{*}$ which extends $\vec{\mu}_{\theta}$ and thus extends $\mu_{\theta}$ also.

E.M.S.-U 
Putting $\tilde{a}_{\theta}=t_{\alpha}$ in (8) and making use of (7) we get

$$
t_{\theta}^{-1} t_{\alpha} t_{\theta}=t_{\alpha} \bar{\mu}_{\theta}=t_{\alpha}
$$

thus $t_{\alpha}$ commutes with every $t_{\theta}$. This completes the proof of the following theorem.

Theorem 2. For the existence of an extension group $G^{*}$ containing $G$ and inner automorphisms $t_{\sigma}$ of $G^{*}$ extending $\mu_{\sigma}$ for every $\sigma \in \Sigma$ such that $t_{\alpha}$ commutes with every $t_{\sigma}$ it is sufficient that relations (2.1)-(2.5) hold.

\section{Special cases}

From Theorem 2, the following are immediate consequences.

Corollary 1. With the previous notation, it is sufficient for the existence of an extension group with the required property that together with (2.5) the relations

$$
A_{\alpha} \cap A_{\theta}=A_{\theta} \cap B_{\alpha}=A_{\alpha} \cap B_{\theta}=B_{\alpha} \cap B_{\theta}=\{e\},
$$

hold for all $\theta(\neq \alpha) \in \Sigma$; where $e$ denotes the unit element of the group.

For then the conditions of Theorem 2 will be trivially satisfied.

Corollary 2. Again, with the same notation, if $A_{\sigma}$ coincides with $B_{\sigma}$ for every $\sigma \in \Sigma$, i.e. if $\mu_{\sigma}$ maps $A_{\sigma}$ onto itself then for the required extension to be effected, it is sufficient together with (2.5) that

for every $\sigma$ in $\Sigma$.

$$
\left(A_{\alpha} \cap A_{\sigma}\right) \mu_{\alpha}=\left(A_{\alpha} \cap A_{\sigma}\right) \mu_{\sigma}=A_{\alpha} \cap A_{\sigma}
$$

\section{REFERENCES}

(1) C. G. Chehata, Commutative extension of partial automorphisms of groups, Proc. Glasgow Math. Assoc., 1 (IV) (1953), 170-181.

(2) G. Higman, B. H. Neumann and H. Neumann, Embedding theorems for groups, J. London Math. Soc., 24 (1949), 247-254.

FACUlTy OF SCIENCE

THE UNIVERSITY

AlEXANDRIA, EgYPT 